\title{
Study on Search Algorithm of Passenger Travel Route in Railway Trans- port Network
}

\author{
Lu Tong ${ }^{*}$, Lei Nie, Gen-Cai Guo and Jin-mei Li \\ School of Traffic and Transportation, Beijing Jiaotong University, 100044, Beijing, China
}

\begin{abstract}
Study on search algorithm of passenger travel route in railway transport network is of great importance in evaluation on railway operation benefit. Based on features of large-scale network and railway passenger transportation organization, the paper firstly innovatively puts forward the generation method of alternative route set in multiple constraint conditions. $K$ short routes is proposed based on layering network to generate the alternative route set of passenger flow and hybrid genetic algorithm to generate the alternative route set of passenger flow in format of origindestination matrix in order to overcome the shortage of traditional Dijkstra algorithm. Then reasonable routes of passenger flow are selected from the alternative route set. Finally, the algorithm by MATLAB programming can search reasonable route set of 589 passenger flows within 2 minutes in the railway network constituted by 521 stations which verifies the convergence of the algorithm.
\end{abstract}

Keywords: Alternative route set, hybrid genetic algorithm, passenger travel route, railway transport network, reasonable route.

\section{INTRODUCTION}

Passenger travel route is composed under the comprehensive orientation of railway transport organization and travel choice in railway transport network. Search algorithm of passenger route is the basic research of optimization of transport network, which is significant for network optimization and evaluation on the performance of network operation. The railway network scale in China is huge and complicated, which increases the difficulty in studying the search algorithm of passenger travel route.

Scholars have made a great number of studies on the problem at present [1]. Studies the optimization on the selection of travel route under the condition of uncertain road network. The perception time and perception probability of travelers are obtained by the nonlinear conversion of practical travel time and the probability value, which takes the maximum of the perception efficiency of passengers as the optimization objective and seeks for the optimal route [2] Takes the reliability factor for passenger transfer when analyzing the travel route of passengers. Passengers always choose the route with the minimum travel cost in the expected transfer reliability. It adopts MSA passenger flow distribution algorithm which integrates $\mathrm{K}$ short route algorithm to simulate the choice behavior of passenger flow with different demand level of for the travel route. Meanwhile, the predication for the train service level of based on the cost is beneficial for evaluation and optimization on the train timetabling [3]. Proposes an improved Dijkstra algorithm to solve the shortest route problem, which includes the setting of two fuzzy parameters: addition principle of two edges and comparison of two routes which cover fuzzy-value edges. It introduces graded integration representation to improve the typical Dijkstra algorithm [4] Puts forward a kind of accurate algorithm for solving the shortest route problem with constraints. This algorithm is based on the idea of network pulse propagation, which manifests that the algorithm is better than the labeling algorithm of the shortest route verified by a road network with 40000 nodes and 80000 edges. The algorithm can solve any problem with column-generation structure, such as the design of road network of BRT and duty scheduling of multiple events. The solution efficiency is obvious superior to the current business linear solution software [5]. Improves the standard simulated annealing algorithm to solve the problem of dynamic route planning, which can get the optimal or approximately optimal route by comparing with classic Dijkstra algorithm [6]. Constructs the train service network based on existing train route and adopts the fast search algorithm to solve the feasible route set introducing the concept of information entropy to endow route weights and choose the relatively superior routes [7]. Constructs dynamic road network model to optimize the evacuation route for vehicles based on Dijkstra algorithm. It demonstrates that the model can provide relatively superior evacuation route for vehicles and provides the decision maker with emergency evacuation strategies under three different cases [8]. Puts forward the search mechanism of the shortest route in the road network with multiple traffic patterns and pays much attention to the attraction of the transfer station of different traffic modes, which encourages more routes pass through the transfer station. The multi-modebased road network endows nodes and edges with different weight value and takes the money expenditure, time consumption and transfer inconvenience in the travel process of passenger flow as the optimization objective to plan the travel route of passenger flow. 
From research status, overseas scholars have made a lot of studies on passenger travel route, which mainly adopts traditional Dijkstra algorithm. Due to the capacity constraint and of other limitations of alternative route set, although traditional Dijkstra algorithm based on mathematical viewpoint can get multiple routes, the solution is always discrepant with the practical situation. Routes may include circuits; many sections are overlapped and used by solved multiple routes, which is hard to demonstrate that they are different selection schemes. In addition, as for multi-mode and multiclass hybrid network, the network scale is huge and complicated; it may not get the optional solution in practical application. If it can get the satisfactory solution in less time and space, the algorithm is feasible.

Based on the above analysis, the paper adopts the idea that reasonable routes of passenger flow is generated from alternative route set to study the efficient search algorithm according to characteristics of the large-scale railway network.

\section{NETWORK TOPOLOGY CONSTRUCTION}

Construction of railway network is the foundation for the optimization of train service network. The network includes station, section, line, city, high-speed train section office, hub, railway administration. Railway network is presented by the diagram where node represents stations and the edge or arc represents the relationship between two adjacent stations which includes linking-up edge in section or hub and virtual line of transfer in two stations in the hub. The quantitative attribute of edge or arc represents the weight value. Different edges of the same node have different attributes so that the node set and edge set compose a weight digraph. Besides, lines which constitute the physical railway network have multiple classes of design speed so that it is called as mixed road network.

The mixed railway network of is abstracted into a weight digraph $G=(N, E)$, where $N$ is the node set, $E$ is edge set; the topologic relation in the physical railway network is presented by the relation between the node and edge in the diagram.

\section{RELATED CONCEPTS OF ROUTE}

\subsection{Physical Route}

In railway network there are many routes of every passenger flow but reasonable routes only account for a small part of all enumeration routes. Therefore, the paper firstly generates alternative route set and then screens the generated reasonable route set according to the rules of railway transport organization as the route set of passenger flow.

\subsubsection{Concept of Alternative Route Set}

Alternative route set which includes most of reasonable routes. Relevant rules and algorithm are introduced to diminish alternative route set by simplifying the basic railway network and select the reasonable routes from the alternative route set.

The alternative route set should conform to the constriction of three basic conditions, including logic search condi- tion of route, train operation route and passenger travel route, where:

A. Basic conditions of logic search of route include: alternative route must be correct route; define $r(i)$ as the shortest distance from origin $O$ to node $i ; s(i)$ is the shortest distance from node $i$ to destination $D . r(i)$ and $s(i)$ is calculated to guarantee their existence.

B. Logic search condition of train operation route: according to the principle of railway transport organization, train operation route is basically composed by railway sections with the same grade of design speed. Existing trains and high-speed trains operate in separated lines, which include the transport organization pattern of off-line highspeed trains or in-line trains in existing line. However, it is impossible for train routes pass by different sections with higher speed and lower speed alternatively for many times in the stage of searching alternative route. Thus, some unreasonable routes are eliminated in the process of route searching according to the principle of train operation route.

C. Logic search condition of passenger travel route: passengers will not choose ring route with repeated sections. Passengers choose the section $e(i, j) \in E$ because the distance is farther to from $i$ to $O$ and closer from $j$ to $D$. Thus, the logic foundation eligible for travel of passengers is that every section $e(i, j)$ meets the condition: $r(i)<r(j), s(i)>s(j)$.

\subsubsection{Concept of Reasonable Route Set}

Reasonable route set which meets some constraint conditions including line speed class, train speed class, transfer frequency and total mileage. Every route has specific advantages compared with other routes.

\subsection{Relevant Concepts of Passenger Travel Route}

Railway passenger travel route is the foundation for determining the direction of every passenger flow and train operation route. The optimization of train origin and destination, train operation route should confirm to the distribution of passenger flow in the railway network.

The selection of passengers for the optimal travel route under the transport capability constraint of various feasible routes, considers many factors from the origin to destination and every passenger flow is distributed in railway lines under the transport capability constraint; passenger travel route is established on physical route and train operation route.

\subsection{Variety of Passenger Travel Route}

Single route: due to characteristics of railway transport organization, routes distributed by railway passenger flow are different from the road transport route. In general case, every passenger flow in railway network only chooses one route namely, the single route, because trains with the same OD have the same operation route. The route with shortest mileage is the shortest route where equivalent value of time, cost and other integrated factors are the rules for selection of passenger flow in physical railway network. 
Multi-route: with the construction of high-speed railway, parallel routes among nodes of railway network increase and there are at least two routes from the origin to destination of passenger flow. Due to transport capability constraint, every passenger flow is distributed in different reasonable routes, which forms multi-route.

Specific route: in general case, the transport department cannot directly assign the passenger flow to feasible travel routes but it can stipulate that some passenger trains run on the specific route, which indirectly affects the selection of passenger route.

\section{GENERATION OF ALTERNATIVE ROUTE SET}

The method of generation of alternative route set mainly includes section deletion, section penalty and $K$ shortest route, etc. The paper adopts the method of $K$ shortest route to generate the alternative route set of passenger flow. Due to capability constraint or other restriction: smaller travel cost, less travel time, maximal transfer frequency. Passenger flow should consider both the shortest route and sub-short route, sub-sub-short route...namely $K$ short route. $K$ short route algorithm can calculate $K$ shortest routes arranged from small to large in length. Its definition varies from different research objects. In railway network, the weight value of edges can refer to distance and the first $K$ shortest route is shortest. It can also refer to time and the time of the first $K$ shortest route is the shortest.

Traditional $K$ shortest routes algorithm is mainly based on mathematic viewpoint, while the solution is always discrepant with the practical situation. For example, routes may include ring circles, many same sections are overlapped and used by solved multiple routes, and many sections are similar, hard to demonstrate that they are different selection schemes. The weight value of each edge in the graph of the basic physical railway network is positive where there exist rings and circles in the network and multiple edges (segmental arc) between two points so that the structure is extremely complicated. As for such large-scale network, in order to solve $K$ shortest route, it needs to design a kind of algorithm with relatively lower complicity and concise thinking, which can be carried out easily. The common algorithm for solving $K$ shortest route is Dijkstra algorithm and the improved algorithm. With the increase of network size, some intelligent heuristic algorithm is gradually applied by scholars.

\subsection{Network Layering}

In railway network, it adopts layered search algorithm to search alternative route due to difference of railway line grade. The basic idea is to divide railway network into multiple layers based on the grade of railway line, which is dividing the overall railway network into multiple subnetworks. Every sub-network is the abstract expression of original railway network. Ignore unnecessary nodes in senior sub-network and the contained physical sections will be consequently reduced. Sub-network node is the subset or subrange of nodes of original railway network. Network layering can reduce the search space of alternative routes.
The construction of network layers can determine the layer level of network based on practical demand, given data scale and network operation mode and the sub-network of high-speed railway is determined as the supreme network layer. Meanwhile, it can be successively divided into subnetwork of existing lines and sub-network of interurban lines. Every divided sub-network must guarantee its connectivity and accessibility.

The idea of network layering is as follows: $G_{i}=\left(N_{i}, E_{i}\right)$ is the sub-network in level $i$, $G_{i}=\left(G_{1}, G_{2}, \ldots, G_{n}\right)=\left(\left(N_{1}, E_{1}\right),\left(N_{2}, E_{2}\right), \ldots,\left(N_{n}, E_{n}\right)\right)$; assuming the network is divided into $M$ layer levels, $K$ short route of the $j^{\text {th }}$ node from the origin $S_{\text {to }} i^{\text {th }}(i<M)$ subnetwork is $D^{k}\left(n_{i j}\right)=S^{k}\left(n_{i-1}\right)+d^{k}\left(n_{i j}\right)$, among which:

$S^{k}\left(n_{i-1}\right)$ : The length (or resistance) of the $k^{\text {th }}$ short route obtained from the first sub-network to the $i-1^{\text {th }}$ subnetwork, where $S^{k}\left(n_{i-1}\right)=\sum_{m=1}^{i-1} d^{k}\left(n_{m}\right)$.

$d^{k}\left(n_{m}\right)$ : The length (or resistance) of the ${ }^{k \text { th }}$ short route in the $m$ th sub-network;

$d^{k}\left(n_{i j}\right)$ : The length (or resistance) of the ${ }^{k}$ th short route of the ${ }^{j \text { th }}$ node in the ${ }^{i \text { th }}$ sub-node;

$S_{i}$ : The nearest connection node of destination, $S$ is in the sub-network in level $i$;

$D_{i}^{\prime}$ : The nearest connection node of destination, $D$ is in the sub-network in level $i$;

$K$ short route of multilayer network can be resolved into the route search of multiple sub-networks and the algorithm is as follows:

Step 1: Searching the $K$ short route from the origin $S$ to $D$ in the sub-network $G_{1}$, the nearest connection node $S_{1}^{\prime}$ from the origin and the nearest connection node $D_{1}^{\prime}$ from the destination $D$; then the route is obtained by route search algorithm: ${ }^{k}{ }_{1 S_{1}^{\prime} D_{1}^{\prime}}=\left(s_{1}^{\prime}, v_{11}, v_{12}, \ldots, v_{1 k^{\prime}}, d_{1}^{\prime}\right)$.

Step 2: Judging whether there is sub-network $G_{2}$. If there is, it finds the nearest overline node $S_{2}^{\prime}$ from the origin $S_{1}^{\prime}$ and the closer overline node $D_{2}^{\prime}$ to the destination $D_{1}^{\prime}$ in $G_{2}$. The route is searched by route search algorithm.

$$
\begin{gathered}
\Pi_{2 s_{2}^{\prime} s_{1}^{\prime}}^{k}=\left(s_{2}^{\prime}, v_{2 s_{1}}, v_{2 s_{2}}, \ldots, v_{2 s k^{\prime}}, s_{1}^{\prime}\right), \\
\Pi_{2 D_{2}^{\prime} D_{1}^{\prime}}^{k}=\left(d_{2}^{\prime}, v_{2 d_{1}}, v_{2 d_{2}}, \ldots, v_{2 d k^{\prime}}, d_{1}^{\prime}\right)
\end{gathered}
$$

Step 3: Merging the route $\Pi_{2 S_{2}^{\prime} D_{2}^{\prime}}^{k}$ in the network $G_{2}$ with the route $\Pi^{k}{ }_{1 S_{1}^{\prime} D_{1}^{\prime}}$ in the sub-network $G_{1}$ and generate the complete route ${ }^{\Pi_{2 S_{2}^{\prime} D_{2}^{\prime}}^{k}}$. 


$$
\begin{aligned}
& \quad \Pi^{k}{ }_{2 S_{2}^{\prime} D_{2}^{\prime}}=\left(\left(s_{2}^{\prime}, v_{2 s 1}, \ldots, v_{2 s k^{\prime}}, s_{1}^{\prime}\right),\left(s_{1}^{\prime}, v_{11}, \ldots, v_{1 k^{\prime}}, d_{1}^{\prime}\right),\right. \\
& \left.\left(d_{1}^{\prime}, v_{2 d k^{\prime}}, \ldots, v_{2 d 1}, d_{2}^{\prime}\right)\right)
\end{aligned}
$$

Step 4: Judging whether there is sub-network ${ }^{G_{i}}$; if there is, continuing to carry out route search and route merger described in Step 2 and Step 3 in the $i_{\text {th sub-network. Until }}$ the sub-network in level $M$ found, the search of optimal route is completed.

\subsection{Solution of K Short Route of Railway Network by Hybrid Genetic Algorithm}

The national-wide railway network is a multi-mode and multi-class network composed by existing line and highspeed lines. The network is extremely complicated and huge including thousands and tens of thousands of station nodes so that it is necessary to concern about the route search in the large-scale network. In practical application, it may not get the optimal solution. If getting the satisfactory solution in smaller time and space is possible, the algorithm is feasible with the practical application value. The paper adopts heuristic algorithm to generate the $K$ short route and designs hybrid genetic algorithm to solve the problem. The algorithm can get convergence in short time and get the satisfactory solution.

\subsubsection{Coding Method of Chromosome}

Coding is to express the feasible solution in the solution space of the problem as genotype string structural data (one feasible solution is mapped into a character string) and the coding method affects the running time of algorithm and size of solution space. Meanwhile, it is also the basis for the design of fitness function.

Set the weight diagraph $G=(N, E)$, where $N$ is the vertex set, $E$ is the edge set, $c(e)$ is the weight value of edge. Natural number is adopted to represent the node in the graph. The genic value of chromosome is the node number in the network and the marshalling sequence of nodes represents the route from node $n_{0} \rightarrow n_{t}$ and automatically chromosome $P=\left(n_{1}, n_{2}, \ldots, n_{t}\right)$ represents the route from node $n_{0} \rightarrow n_{t}$ in the graph. Different routes pass through different nodes so that the length of chromosome is variable. If numbering nodes are passed by route from node 1 to node 20 as ${ }_{1} \rightarrow_{2} \rightarrow_{4} \rightarrow_{11} \rightarrow_{17} \rightarrow_{19} \rightarrow_{20}$, the code of chromosome is 12411171920 .

\subsubsection{Initial Population}

The entire population refers to the solution of a problem and the quantity of chromosomes in the population is uncertain, which mainly depends on the number of routes in the population. The paper adopts the method of randomly increasing a chromosome to randomly increase the population. If there are $k$ routes in current solution and $k+1$ chromosomes in the population, randomly initiate the population.

\subsubsection{Genetic Operator}

(1) Choice: Two different chromosomes are randomly selected from the population as two parent chromosomes, $P 1$ and $P 2$.

(2) Exchange: $n$ continuous gene locus is selected from parent chromosomes $P 1$ and $P 2$, then are exchanged. $n$ is the randomly generated integer and the value of $n$ is smaller than the length of chromosome. For example, $n=4$, the exchange process is executed as:

$\left\{\begin{array}{l}P 1: 12|4111719| 20 \\ P 2: 35|68910| 21\end{array} \Rightarrow\left\{\begin{array}{l}Q 1: 12|68910| 20 \\ Q 2: 35|4111719| 21\end{array}\right.\right.$

(3) Movement: $m^{\text {th }}$ gene locus from parent chromosome $P 1$ and $m^{\prime \text { th }}$ from $P 2$ is randomly selected. The value of $m$ and $m^{\prime}$ must be smaller than the length of parent chromosome. For example, $m=4, m^{\prime}=3$, the movement process is as:

$\left\{\begin{array}{l}P 1: 12|4579| 20 \\ P 2: 361115\end{array} \Rightarrow\left\{\begin{array}{l}Q 1: 1220 \\ Q 2: 36|4579| 1115\end{array}\right.\right.$

(4) Crossover: Crossover operation means a pair of parent chromosomes generates the child chromosomes by exchanging part of genes. The method of crossover mainly includes single-point crossover, multi-point crossover and uniform crossover. Adopt PMX (Partially Matched Crossover) and the specific crossover method is as follows:

\section{(1) Determination of crossover point}

It selects a gene locus from parent chromosomes, $P 1$ and $P 2$ respectively and takes these two gene locus as the crossover point. The crossover point is taken as the origin to exchange the gene locus after the crossover of two parent chromosomes to generate child chromosome.

$\left\{\begin{array}{l}P 1: 124 \mid 57920 \\ P 2: 36 \mid 5791115\end{array} \Rightarrow\left\{\begin{array}{l}Q 1: 124 \mid 5791115 \\ Q 2: 36 \mid 57920\end{array}\right.\right.$

Assuming that the crossover point is the gene locus 4 in parent chromosome $P 1,5$ in parent chromosome $P 2$, genes after gene locus 4 in $P 1$ and genes after gene locus 6 in $P 2$ are exchanged to generate new individuals. If the gene locus for reciprocal exchange cannot be connected with each other, the gene locus is determined repeatedly which can be connected backwards according to the sequence of gene locus. If both of them have no genes which can be connected, the crossover of two parent chromosomes $P 1$ and $P 2$ is unsuccessful. Other parent chromosomes are anew selected to make crossover.

\section{(2) Coverage of duplicate genes}

In child chromosomes generated by crossover, if there are duplicate genes, the covering method is adopted to eliminate the same genes, which represents the elimination of ring circles. For example, the child chromosome generated after the crossover of parent chromosome is:

$$
Q 1: 245691151926 \text {; }
$$


It can be seen that there is duplicate gene 5 in the child chromosome generated after crossover. Genes between the duplicate gene 5 are eliminated to get the new child chromosome is: $Q 1: 2451926$

(5) Mutation operator: Basic mutation operator is used to generate new gene individual.

The paper adopts basic mutation operator to randomly designate several gene locus by mutation probability to make mutation operation on parent chromosome and exchange two gene element locus to generate new chromosome, which makes the population diverse. It adopts chromosome to represent the route so that mutation operator can take the route section composed by nodes as the gene block to achieve the mutation of gene block. Duplicate genes generated in chromosomes after mutation can be eliminated.

(6) Reservation operator of the optimal gene individual

The optimal individual will be obtained in the update process of population reproduction. In order to avoid the damage from crossover and mutation operator, the optimal gene individual is saved to reserve the operator. The specific operation is copying the optimal individual completely before the operation of crossover and mutation, until there is better individual.

\section{(7) Update operation}

The ordering method is adopted to choose new population. The formula is proposed to undertake the update operation of every generation of chromosome,

$$
Q^{\prime}(i+1)=\operatorname{Rank}(Q(i)+J(i+1)+B(i+1)) \text {, where } Q \text { is the }
$$
newly generated individual, $Q$ refers to update of the previous generation of chromosome population; $J$ is the chromosome after crossover operation and $B$ is the chromosome after mutation operation.

(8) Fitness function and annealing constraint processing technology

There are two methods for genetic algorithm to solve the constrained optimization problem. (1) If the number of nonfeasible solution in the chromosome population is less than that of feasible solution, it can exclude the non-feasible solution and only reverse the feasible solution. (2) If the nonfeasible solution in the chromosome population accounts for a large proportion, it is appropriate to adopt penalty function method, imposing penalty on chromosome individual which violates the constraint condition. When solving the problem of $K$ short route in psychical railway network, non-feasible solution accounts for a large proportion so that it adopts the penalty function method.

The key issue of penalty function method is setting the penalty term. In order to overcome the shortage that the setting of penalty term is irrelevant with the problem, the paper adopts annealing penalty function method to control the penalty value of non-feasible solution by dynamic adjustment of the temperature. The specific expression is as follows:

$$
F(x)=e^{-M / T} f(x)
$$

$F(x)$ : Fitness of chromosome $x$

$e^{-M / T}:$ Penalty term

$f(x)$ : Objective function value of $x$

$M$ : Constant positive number which is not satisfied with constraint condition (When satisfying the constraint condition, $M=0$ )

\section{$T:$ Temperature}

At the beginning of algorithm, $T$ is larger and $M$ is small so that the penalty value of non-feasible solution is small with week constraint condition to improve the ergodicity of algorithm. With the increase of $T$ value, the fitness value of solution which is not satisfied with the constraint condition is reduced. Such kind of individual does not occur in the evolutionary group. As for non-feasible solution, when $M=0$, no matter how $T$ changes, the fitness function is equal to objective function.

The definition of fitness function is:

$F(x)=e^{-M / T} \frac{1}{f(x)}, T(n)=\alpha T(n-1) \quad(0<\alpha<1)$

When it satisfies the constraint condition, $M=0$.

Otherwise, $M=0$. In the formula, $n$ is the number of iteration; $\alpha$ is a constant. From the formula, it can be seen that the fitness function value is positive. The fitness of route which satisfies the constraint condition is larger. On the contrary, the fitness of route which does not satisfy the constraint condition is relatively smaller.

Specific steps of hybrid genetic algorithm for solving $\mathrm{K}$ short route of railway network are shown in Fig. (1).

The relationship between hybrid genetic algorithm for solving $\mathrm{K}$ short route of railway network and the concept of biogenetics are shown in Table $\mathbf{1 .}$

\section{GENERATION OF REASONABLE ROUTE SET}

In the field of urban public transport network optimization, it usually adopts the method of $K$ short route to find the reasonable route. However, such method is just based on the mathematic perspective not combining with characteristics of railway transport network. For example, it does not take the specific route between stations and transfer into consideration and it cannot be directly applied in the search of passenger travel route of railway network. The paper adopts the method of generating reasonable route set from alternative route set.

After the generation of alternative set by $K$ shortest algorithm, it needs to make re-optimization on alternative route because not every alternative route is finally selected as the reasonable route. Reasonable route set stresses every route has diversity compared with other routes. It should choose the reasonable route from alternative route set according to practical demand. 


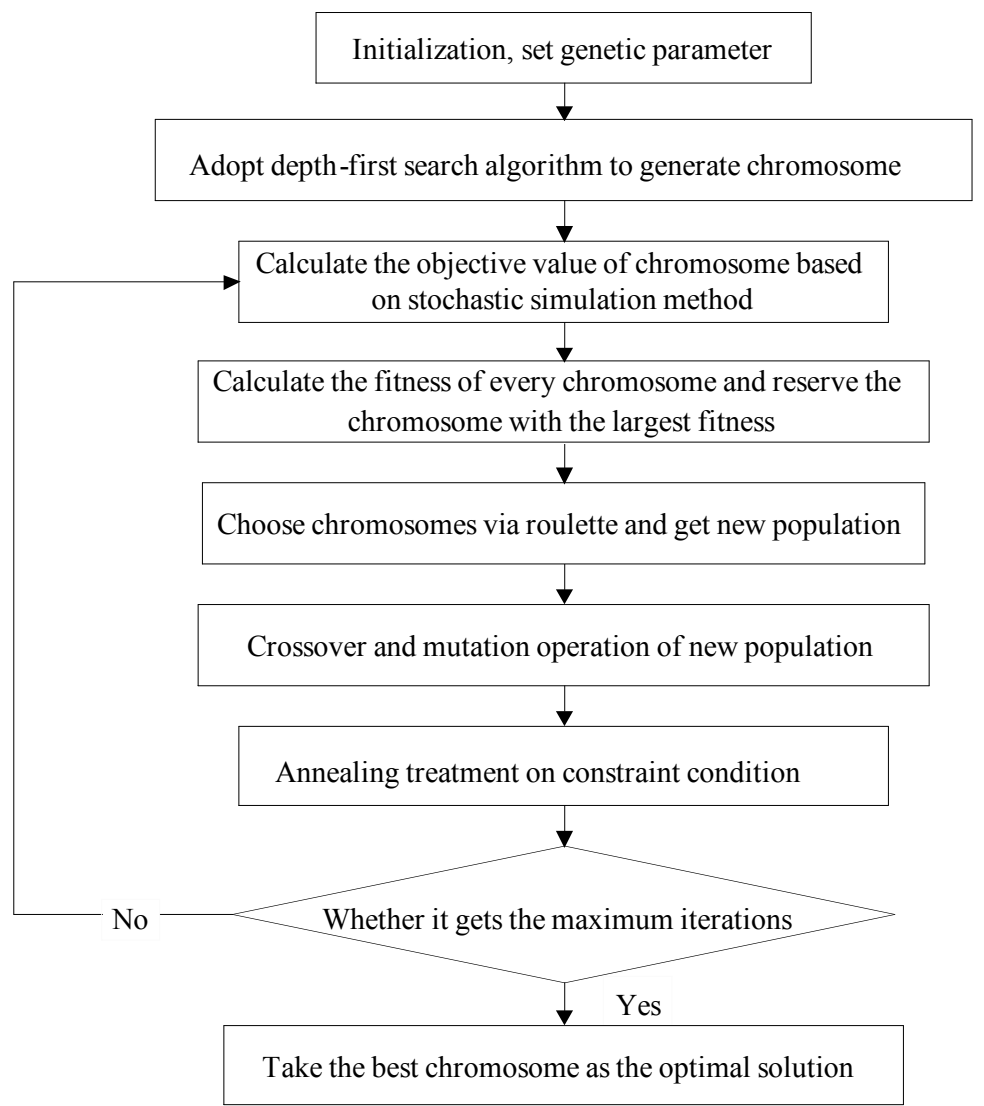

Fig. (1). Hybrid genetic algorithm.

Table 1. The relationship between hybrid genetic algorithm and the concept of biogenetics.

\begin{tabular}{|c|c|c|}
\hline Concept of Biogenetics & $\begin{array}{c}\text { Hybrid Genetic Algorithm for Solving K Short Route of } \\
\text { Railway Network }\end{array}$ & Function of Genetic Algorithm \\
\hline Survival of the fittest & K optimal routes & $\begin{array}{c}\text { When the algorithm stops, the solution of optimal objective } \\
\text { value is much probably reserved. }\end{array}$ \\
\hline Individual & One feasible route set & Solution of the problem \\
\hline Chromosome & $\begin{array}{l}\text { The marshalling sequence of nodes of routes; routes pass } \\
\text { through different nodes so that the length of chromosome is } \\
\text { different. }\end{array}$ & Code of solution (character string, vector, etc) \\
\hline Gene & $\begin{array}{l}\text { Node number in network (natural number represents the node } \\
\text { in the graph) }\end{array}$ & $\begin{array}{l}\text { Characteristics of every component in the solution (such as } \\
\text { the values of various components) }\end{array}$ \\
\hline Fitness & In direct proportion to objective function value & Fitness function value \\
\hline Group & Feasible route set & $\begin{array}{l}\text { A set of selected solution (the number of solution is the size } \\
\text { of the group) }\end{array}$ \\
\hline Population & Initial population (initial solution of the problem) & a set of solution selected according to the fitness function \\
\hline Mating & $\begin{array}{l}\text { two different chromosomes (routes) are selected and their route } \\
\text { sections are exchanged }\end{array}$ & $\begin{array}{c}\text { The process of generating a set of new solution based on } \\
\text { the mating principle }\end{array}$ \\
\hline Mutation & $\begin{array}{c}\text { some node or several nodes are randomly selected to make } \\
\text { mutation }\end{array}$ & The change process of some component of the code \\
\hline
\end{tabular}




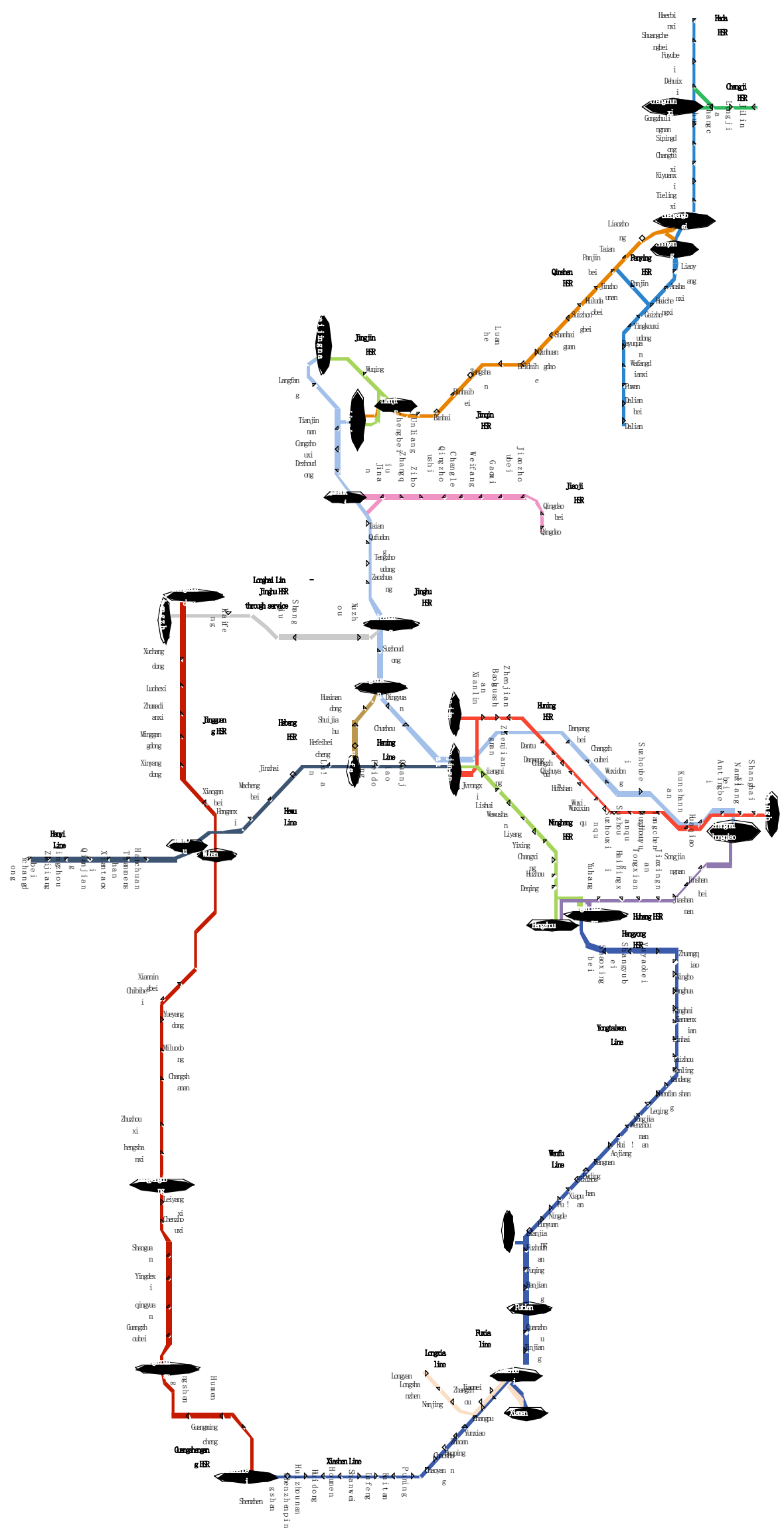

Fig. (2). The city nodes in relevant railway network of Beijing-Shanghai high-speed railway. 
Many factors have effect on reasonable routes. In practical application, it is necessary to consider several main factors. For example, when choosing the route of physical railway network, the overall length, total running time, operation frequency, percentage of total running time (distance) in high-speed line, percentage of the minimum running time (distance) in single-section of high-speed line, percentage of the longest running time (distance) in single-section of highspeed line and attribute of station. These factors will affect the choice of reasonable route from alternative route set.

In addition, the choice of reasonable route also needs to consider the factor of politics and economy. In practical application, some cities or stations need indispensable train services on the behalf of specific routes. However, when adopting $K$ short route to solve the alternative route set, there are no specific routes in the set so that some designated specific routes must be added in the reasonable route set to get the complete reasonable route set.

\section{CASE ANALYSIS}

\subsection{Construction of Railway Network of Beijing- Shanghai High-Speed Railway}

The long-term railway network related with BeijingShanghai high-speed railway is constructed with elementary database, including line name, design speed, designed traffic capacity of lines (ten thousand person-time/year), running date of line, name of stations $\mathrm{n}$ the origin section, located cities of the stations in the origin section, name of stations in the destination of section, located cities of the stations in the destination section, section distance. The design speed of the related railway lines must be larger than $250 \mathrm{~km} / \mathrm{h}$.
City nodes related to the railway network of BeijingShanghai high-speed railway are selected. Statistics are gained including the running route of trains and trains related to existing lines (including trains in existing lines, with oneend cross existing lines, with both ends cross existing lines) of Beijing-Shanghai. According to forecast passenger flow demand of high-speed railway in the long-term railway network, the current operation situation of trains in existing lines is analyzed and the ODs of these trains are recorded. The paper chooses 601 railway sections from 41 main cities to constitute relevant railway network of long-term BeijingShanghai high-speed railway. The network covers 50 passenger railway lines, 17 intercity lines and 1 BeijingShanghai high-speed railway line. The city nodes of related railway lines show in Fig. (2).

\subsection{Generation of Reasonable Route Set}

The hybrid genetic algorithm is adopted to solve K short route in the network to optimize the alternative route. Values of main parameters are: number of gene: GAnum=10; mutation probability: mutation $\mathrm{P}=0.5$; update probability: update $\mathrm{P}=0.5$

After the optimization of alternative route, the reasonable route set is generated. Fig. (3) shows the changing curve of fitness value for 100 times of iteration of the algorithm. The solution is obtained in smaller time and space, shown in Table 2 .

\section{CONCLUSION}

The paper proposes the method of $K$ short route to generate the alternative route set of passenger flow and designs

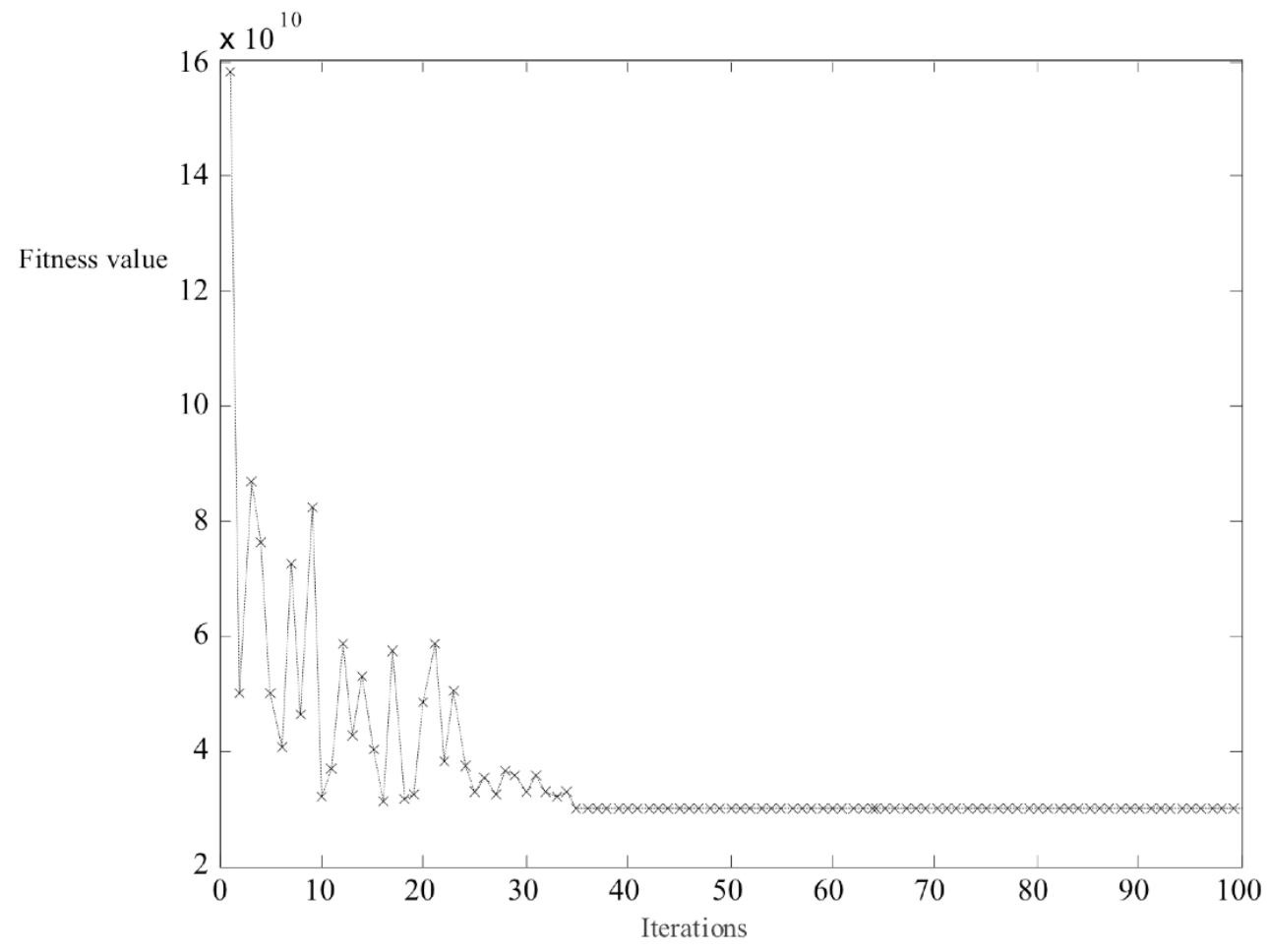

Fig. (3). Iteration curve of fitness value. 
Table 2. Reasonable route set of passenger flow in part of $O-D$.

\begin{tabular}{|l|l|}
\hline \multicolumn{1}{|c|}{ Name of O-D } & \multicolumn{1}{c|}{ Passenger Travel Route (Station Node) and Distance } \\
\hline \hline \multirow{3}{*}{ Beijing-Langfang } & (1) Beijing South $\rightarrow$ Langfang 59.50 \\
& (2) Beijing $\rightarrow$ Beijing South $\rightarrow$ Langfang 69.50 \\
& (3) Beijing West $\rightarrow$ Beijing South $\rightarrow$ Langfang 74.50
\end{tabular}

hybrid genetic algorithm to generate the alternative route set in order to overcome the shortage of traditional algorithm. It puts forward the method of generating reasonable route set from the alternative route set according to the characteristics of railway transport network. Finally, it verifies the convergence of algorithm in the context of large-scale railway network. The proposed algorithm is significant for guiding the practical application.

\section{CONFLICT OF INTEREST}

The authors confirm that this article content has no conflict of interest.

\section{ACKNOWLEDGEMENTS}

This research is supported by Railway ministry science and technology management project (2013X014-C), Fundamental Research Funds for the Central Universities (Beijing Jiaotong University) under Grant (2014JBZ008) and evaluation of High-Speed Railway Passenger Train Service Plan and System Development (2014X010-A).

\section{REFERENCES}

[1] R. D. Connors, and A. Sumalee, "A network equilibrium model with travellers' perception of stochastic travel times," Transportation Research Part B: Methodological, vol. 43, no. 6, pp. 614-624, 2009.

[2] F. Shi, Z. Zhou, J. Yao, and H. Huang "Incorporating transfer reliability into equilibrium analysis of railway passenger flow," European Journal of Operational Research, vol. 220, pp. 378-385, 2012.

[3] D. Yong, Y. Chen, Y. Zhanga, and S. Mahadevanc, "Fuzzy Dijkstra algorithm for shortest path problem under uncertain environment," Applied Soft Computing, vol. 12, pp. 1231-1237, 2012.

[4] L. Lozano, and A. L. Medaglia, "On an exact method for the constrained shortest path problem," Computers \& Operations Research, vol. 40, pp. 378-384, 2013.

[5] M. Hui, and Y. C. Tian, "Dynamic robot path planning using an enhanced simulated annealing approach," Applied Mathematics and Computation, vol. 222, pp. 420-437, 2013.

[6] X. Yang, L. Liu, Y. Li, and R. He "Route Selection for Railway Passengers: A Multi-objective Model and Optimization Algorithm," Journal Of Transportation Systems Engineering And Information Technology, vol. 13, no. 5, pp. 72-78, 2013.

[7] Y. Chen, S. Shen, T. Chen, and R. Yang, "Path Optimization Study for Vehicles Evacuation Based on Dijkstra algorithm," Procedia Engineering, vol. 71, pp. 159-165, 2014.

[8] D. Ambrosino, and A. Sciomache, "An algorithmic framework for computing shortest routes in urban multimodal networks with different criteria," Procedia Social and Behavioral Sciences, vol. 108, pp. 139-152, 2014.

(C) Tong et al.; Licensee Bentham Open.

This is an open access article licensed under the terms of the (https://creativecommons.org/licenses/by/4.0/legalcode), which permits unrestricted, noncommercial use, distribution and reproduction in any medium, provided the work is properly cited. 